\title{
Article \\ Differentiated Thyroid Cancer with Biochemical Incomplete Response: Clinico-Pathological Characteristics and Long Term Disease Outcomes
}

\author{
Miriam Steinschneider ${ }^{1,2}$, Jacob Pitaro ${ }^{2,3}$, Shlomit Koren ${ }^{1,2}$, Yuval Mizrakli ${ }^{2,3}$ (D), Carlos Benbassat ${ }^{1,2}$ \\ and Limor Muallem Kalmovich 2,3,* \\ 1 Endocrine Institute, Shamir (Assaf Harofeh) Medical Center, Zerifin 7030000, Israel; \\ miriamsh@shamir.gov.il (M.S.); shlomitks@shamir.gov.il (S.K.); benbassatc@shamir.gov.il (C.B.) \\ 2 Sackler Faculty of Medicine, Tel-Aviv University, Tel-Aviv 6997801, Israel; pitaroj@shamir.gov.il (J.P.); \\ YuvalMi@shamir.gov.il (Y.M.) \\ 3 Department of Otolaryngology-Head and Neck Surgery, Shamir (Assaf Harofeh) Medical Center, \\ Zerifin 7030000, Israel \\ * Correspondence: LimorMK@Shamir.gov.il
}

\section{check for}

updates

Citation: Steinschneider, M.; Pitaro, J.; Koren, S.; Mizrakli, Y.; Benbassat,

C.; Muallem Kalmovich, L.

Differentiated Thyroid Cancer with

Biochemical Incomplete Response:

Clinico-Pathological Characteristics and Long Term Disease Outcomes. Cancers 2021, 13, 5422. https:// doi.org/10.3390/cancers13215422

Academic Editors: Kennichi Kakudo and Vasyl Vasko

Received: 15 August 2021

Accepted: 26 October 2021

Published: 29 October 2021

Publisher's Note: MDPI stays neutral with regard to jurisdictional claims in published maps and institutional affiliations.

Copyright: (C) 2021 by the authors. Licensee MDPI, Basel, Switzerland. This article is an open access article distributed under the terms and conditions of the Creative Commons Attribution (CC BY) license (https:// creativecommons.org/licenses/by/ $4.0 /)$.
Simple Summary: Biochemical incomplete response (BIR) is defined as elevated thyroglobulin or rising thyroglobulin antibodies following treatment without structural evidence of disease at 1-2 years after initial treatment. The long-term outcome of such patients is still poorly characterized, with some progressing to structural disease, while others maintain BIR for decades or revert to non-evidence of disease (NED). In this study, we aimed to identify factors that could predict any of the above possible outcomes. In our cohort of 83 BIR patients with a mean follow-up of 12 years, $41 \%$ progressed to structural disease. Of them, $11.8 \%$ remained BIR, and 38.2\% reverted to NED.

Abstract: Although most patients with differentiated thyroid cancer (DTC) and biochemical incomplete response (BIR) follow a good clinical outcome, progression to structural disease may occur in $8-17 \%$ of patients. We aimed to identify factors that could predict the long-term outcomes of BIR patients. To this end, we conducted a retrospective review study of 1049 charts from our Differential Thyroid Cancer registry of patients who were initially treated with total thyroidectomy between 1962 and 2019. BIR was defined as suppressed thyroglobulin $(\mathrm{Tg})>1 \mathrm{ng} / \mathrm{mL}$, stimulated $\mathrm{Tg}>10 \mathrm{ng} / \mathrm{mL}$ or rising anti-Tg antibodies, who did not have structural evidence of disease, and who were assessed 12-24 months after initial treatment. We found 83 patients $(7.9 \%)$ matching the definition of BIR. During a mean follow-up of $12 \pm 6.6$ years, 49 (59\%) patients remained in a state of BIR or reverted to no evidence of disease, while $34(41 \%)$ progressed to structural disease. At the last follow-up, three cases (3.6\%) were recorded as disease-related death. The American Thyroid Association (ATA) Initial Risk Stratification system and/or AJCC/TNM (8th ed.) staging system at diagnosis predicted the shift from BIR to structural disease, irrespective of their postoperative Tg levels. We conclude that albeit $41 \%$ of BIR patients may shift to structural disease, and most have a rather indolent disease. Specific new individual data enable the Response to Therapy reclassification to become a dynamic system to allow for the better management of BIR patients in the long term.

Keywords: papillary thyroid cancer; biochemical incomplete response; thyroglobulin; prognosis; survival

\section{Introduction}

The initial assessment of patients with differentiated thyroid cancer (DTC) is based on AJCC/TNM staging for predicting disease-specific mortality and the American Thyroid Association (ATA) risk stratification criteria to predict the risk of persistent or recurrent disease-both are static risk estimates that are obtained immediately after primary therapy [1]. Dynamic risk stratification (DRS) of DTC patients that takes into consideration 
the response to initial treatment being reassessed at 1-2 years was introduced by Tuttle et al. [2] and revealed significant shifts in the risk categories of DTC patients. Not without a word of caution, this system was adopted by the ATA 2015 guidelines [1], as it does provide a more individualized approach to medical management and a better approach for the prediction of disease recurrence. Response to treatment is described by four categories, including excellent response (ER), biochemical incomplete response (BIR), structural incomplete response (SIR), and intermediate response (IR). These categories are based on post-treatment $\mathrm{Tg}$ and anti-Tg levels during follow-up and on imaging studies performed during follow-up. The BIR category is defined as persistent abnormal suppressed and/or stimulated $\mathrm{Tg}$ values or elevated anti-Tg antibodies $(\mathrm{TgAb})$ without evidence of structural disease [2]. The BIR group is not uncommon and is seen in 11-22\% of DTC patients [1], and while up to $17 \%$ of them might develop structural disease, $56-68 \%$ will have no evidence of disease (NED) at the last follow up [3]. In this study we aimed to evaluate factors associated with long-term clinical outcome and predictors of structural recurrence in BIR patients.

\section{Materials and Methods}

\subsection{Subjects and Study Protocol}

Patients with DTC who had undergone total thyroidectomy at the department of Otolaryngology-Head and Neck Surgery or its affiliated clinics between 1962 and 2019 (91.4\% after 1990) were included. We evaluated the ATA adopted response to treatment criteria in 1049 patients from our local DTC registry who had enough available data for analysis. A total of 795 patients $(75.8 \%)$ had ER, 91 (8.7\%) had BIR, 139 (13.2\%) had SIR, and $24(2.3 \%)$ had IR. We defined BIR according to the ATA criteria: $\mathrm{sTg}>1 \mathrm{ng} / \mathrm{mL}$, $\mathrm{sTg}>10 \mathrm{ng} / \mathrm{mL}$, or rising TgAb in the absence of any evidence of structural disease when assessed at 1-2 years after initial treatment [1]. The clinical features, medical management, and disease outcome of 83 BIR patients were evaluated throughout a mean follow up period of $12 \pm 6.6$ years. BIR/cure and BIR shift to SIR (BIR-to-SIR) groups were compared for demographic and clinicopathological data, risk factors, medical history, extent of disease, primary treatment, additional treatments, and disease outcome. The following variables were collected: age, sex, pathology (histologic type or subtype: PTC, FTC, Hurtle cell, Tall cell variant, Sclerosing variant, and Insular variant of PTC), TNM stage, ATA risk stratification, laboratory tests, surgery, focality, laterality, lymphocytic infiltration, extrathyroidal extension (ETE), $\mathrm{I}^{131}$ treatment, metastases, response to initial treatment, persistent/recurrent disease, disease-free progression, and overall survival. Multifocal disease was defined as more than one tumor foci in one or both lobes. Persistent disease was defined as any evidence of disease based on sonographic and cytology findings, $\mathrm{Tg}$ levels, and iodine uptake beyond the thyroid bed in a diagnostic whole-body scan (DxWBS) assessed at 1-2 years after primary treatment. Patients with undetectable stimulated stTg levels upon withdrawal or after recombinant human Thyrotropin (rhTSH), normal findings on neck ultrasound, and a negative DxWBS were defined as disease-free. Recurrent disease was considered in patients who developed any evidence of disease after achieving an excellent response to initial treatment. The 8th edition of the AAJC/TNM system was used for staging. Serum $\mathrm{TSH}$, free $\mathrm{T} 4$, free $\mathrm{T} 3, \mathrm{Tg}$, and $\mathrm{TgAb}$ were measured by means of a chemiluminescence assay (Immulite, 2000; Diagnostic Products Corporation, Los Angeles, CA, USA). The functional and analytical sensitivities were $0.9 \mathrm{ng} / \mathrm{mL}$ and $0.2 \mathrm{ng} / \mathrm{mL}$, respectively.

\subsection{Statistical Methods}

Categorical variables were presented as numbers and percentages and normally distributed continuous variables as mean and standard deviation, with skewed data being presented as median and the Inter-Quartile range. Data normality was assessed using normal plot and the Shapiro-Wilk test. For differences between groups, we used the independent sample t-test or the Mann-Whitney U test and the chi-square or Fisher's exact test for continuous and categorical variables as appropriate. Differences on survival 
and time to recurrence were analyzed using the log-rank test or Cox regression. Since the progression to structural disease was the main outcome of the study, for statistical analysis, patients with persistent BIR and those who reverted to ER were merged as a single group. A $p$-value of less than 0.05 was considered significant. All computations were performed using SPSS version 24 (IBM Corp., Armonk, NY, USA).

\section{Results}

Eighty-three patients with BIR were included in our study, all of whom had undergone total thyroidectomy and $96.4 \%$ of whom had received $\mathrm{I}^{131}$ after surgery. At the last followup, 47 patients $(56.6 \%)$ achieved ER, $19(22.9 \%)$ remained BIR, and out of the 34 patients who had progressed to SIR (BIR-to-SIR), 13 reverted to ER, 4 to reverted BIR, and only 17 remained SIR after additional treatment. Table 1 shows the clinical characteristics of the BIR patients and the comparison between the BIR and SIR-to-BIR groups, where no significant differences were found.

Table 1. Patient demographics and clinical characteristics.

\begin{tabular}{lcccc}
\hline \multicolumn{1}{c}{ Variable } & All & BIR-to-SIR & BIR/Cure & $p$ Value \\
\hline$n$ & 83 & 34 & 49 & \\
Age, mean \pm SD & $47.0 \pm 16.4$ & $48.3 \pm 16.7$ & $46.2 \pm 16.2$ & 0.237 \\
Female, sex $n(\%)$ & $58(69.9 \%)$ & $22(64.7 \%)$ & $36(73.5 \%)$ & 0.355 \\
\hline Thyroid function pre-op, $n(\%)$ & & & & \\
$\quad$ Euthyroid & $72(87.8 \%)$ & $30(90.9 \%)$ & $42(85.7 \%)$ & 0.650 \\
$\quad$ Hypothyroidism & $5(6.1 \%)$ & $2(6.1 \%)$ & $3(6.1 \%)$ & \\
$\quad$ Hyperthyroidism & $5(6.1 \%)$ & $1(3.0 \%)$ & $4(8.2 \%)$ & \\
\hline Hashimoto thyroiditis, $n(\%)$ & $27(33.3 \%)$ & $10(31.3 \%)$ & $17(34.7 \%)$ & 0.658 \\
Familial history, $n(\%)$ & $6(7.2 \%)$ & $3(8.8 \%)$ & $3(6.1 \%)$ & 0.574 \\
Past radiation exposure, $n(\%)$ & $8(9.6 \%)$ & $5(14.7 \%)$ & $3(6.1 \%)$ & 0.157 \\
\hline
\end{tabular}

SIR, structural incomplete response; BIR, biochemical incomplete response.

Data on the tumor characteristics of BIR patients and their primary treatment are shown in Table 2. Neck dissection was performed during total thyroidectomy in $50.6 \%$, of patients, with no differences being found between the BIR-to-SIR and BIR/cure groups. Additionally, there were no significant differences in terms of the histopathological subtypes or the rates of multifocal disease, extrathyroidal extension, and vascular invasion. However, the BIR-to-SIR group presented with statistically significantly bigger primary tumors $(p=0.003)$ and higher T stages $(p=0.007)$ and a nearly significant higher rate of lymph node $(\mathrm{LN})$ involvement $(p=0.066)$. Altogether, the BIR-to-SIR group presented with a significantly higher TNM stage and ATA risk category compared to the BIR patients who did not shift to SIR $(p<0.001)$. Comparing PTC to the FTC BIR patients, no significant differences were found in terms of the clinico-pathological parameters.

For BIR patients, stimulated post-op and last visit Tg levels were $12.3 \pm 14$ and $14.4 \pm 28 \mathrm{ng} / \mathrm{mL}$, respectively; suppressed post-op and last visit Tg levels were $3.9 \pm 11.4$ and $4.7 \pm 15 \mathrm{ng} / \mathrm{mL}$, respectively $(p=\mathrm{ns})$. The comparison of $\mathrm{Tg}$ levels between the BIR and BIR-to-SIR groups showed non-significant differences, with post-op and last visit stTg levels of $8.7 \pm 8$ vs. $19.6 \pm 20 \mathrm{ng} / \mathrm{mL}$ and $9.1 \pm 15$ vs. $27 \pm 46 \mathrm{ng} / \mathrm{mL}$ and postop and last visit-suppressed Tg levels of $4.7 \pm 13$ vs. $1.8 \pm 1.8 \mathrm{ng} / \mathrm{mL}$ and $5.1 \pm 17$ vs. $3.9 \pm 7.3 \mathrm{ng} / \mathrm{mL}$, respectively $(p=\mathrm{ns})$. Even more so, a comparison of the Tg levels at the last follow-up of the BIR-to-SIR patients who reverted to ER with those who remained SIR showed non-significant differences, with sTg levels of $0.04 \pm 0.09$ vs. $13.7 \pm 7.5$ and stTg levels of $1.0 \pm 6.6$ vs. $78.5 \pm 51.6(p=\mathrm{ns})$. 
Table 2. Tumor characteristics.

\begin{tabular}{|c|c|c|c|c|}
\hline Variable & All & BIR-to-SIR & BIR/Cure & $p$ Value \\
\hline$n$ & 83 & 34 & 49 & \\
\hline Neck dissection, $n(\%)$ & $42(50.6 \%)$ & $17(50.0 \%)$ & $25(51.0 \%)$ & 0.593 \\
\hline Post-op I ${ }^{131}$ treatment, $n(\%)$ & $80(96.4 \%)$ & $34(100 \%)$ & $46(93.9 \%)$ & 0.359 \\
\hline $\mathrm{I}^{131}$ initial dose, median (mCi) & $150(80-150)$ & $150(100-150)$ & $100(65-150)$ & 0.028 \\
\hline \multicolumn{5}{|l|}{ Pathological subtype, $n(\%)$} \\
\hline PTC & $64(77.1 \%)$ & $25(73.5 \%)$ & $39(79.6 \%)$ & \multirow{3}{*}{0.474} \\
\hline FTC & $14(16.9 \%)$ & $6(17.6 \%)$ & $8(16.3 \%)$ & \\
\hline Other * & $5(6.0 \%)$ & $3(8.8 \%)$ & $2(4.1 \%)$ & \\
\hline Multifocal, $n(\%)$ & $49(59.0 \%)$ & $21(61.8 \%)$ & $28(57.1 \%)$ & 0.971 \\
\hline Bilateral, $n(\%)$ & $32(39.5 \%)$ & $15(45.5 \%)$ & $17(35.4 \%)$ & 0.585 \\
\hline Tumor size, median (mm) & $18(12,28)$ & $20(14,40)$ & $15(11,24)$ & 0.003 \\
\hline \multicolumn{5}{|l|}{ T stage, $n(\%)$} \\
\hline 1 & $51(63.0 \%)$ & $18(52.9 \%)$ & $33(70.2 \%)$ & \multirow{4}{*}{0.017} \\
\hline 2 & $16(19.8 \%)$ & $6(17.6 \%)$ & $10(21.3 \%)$ & \\
\hline 3 & $11(13.6 \%)$ & $8(23.5 \%)$ & $3(6.4 \%)$ & \\
\hline 4 & $3(3.7 \%)$ & $2(5.9 \%)$ & $1(2.1 \%)$ & \\
\hline Extrathyroidal extension, $n(\%)$ & $18(21.7 \%)$ & $10(29.4 \%)$ & $8(16.3 \%)$ & 0.201 \\
\hline Muscle extension $n(\%)$ & $7(8.9 \%)$ & $4(11.8 \%)$ & $3(6.7 \%)$ & 0.385 \\
\hline Blood vessel involvement $n(\%)$ & $7(8.5 \%)$ & $4(11.8 \%)$ & $3(6.3 \%)$ & 0.404 \\
\hline LN involvement, $n(\%)$ & $36(43.4 \%)$ & $18(52.9 \%)$ & $18(36.7 \%)$ & 0.066 \\
\hline Extra LN extension, $n(\%)$ & $1(1.2 \%)$ & $0(0 \%)$ & $1(2.0 \%)$ & 0.541 \\
\hline \multicolumn{5}{|l|}{ TNM stage, $n(\%)$} \\
\hline 1 & $68(84.0 \%)$ & $25(73.5 \%)$ & $43(91.5 \%)$ & \multirow{3}{*}{0.001} \\
\hline 2 & $11(13.6 \%)$ & $8(23.5 \%)$ & $3(6.4 \%)$ & \\
\hline 3 & $2(2.5 \%)$ & $1(2.9 \%)$ & $1(2.1 \%)$ & \\
\hline \multicolumn{5}{|l|}{ ATA risk assessment, $n(\%)$} \\
\hline Low & $59(72.8 \%)$ & $19(55.9 \%)$ & $40(85.1 \%)$ & \multirow{3}{*}{$<0.001$} \\
\hline Intermediate & $15(18.5 \%)$ & $10(29.4 \%)$ & $5(10.6 \%)$ & \\
\hline High & $7(8.6 \%)$ & $5(14.7 \%)$ & $2(4.3 \%)$ & \\
\hline
\end{tabular}

SIR, structural incomplete response; BIR, biochemical incomplete response; PTC, papillary thyroid cancer; FTC follicular thyroid cancer; ATA, American Thyroid Association; LN, lymph nodes. * Other-PTC variants including Insular, Tall cell, Sclerosing; Hurtle cell carcinoma.

Table 3 shows the additional treatments and disease outcomes observed during followup. Post operative I ${ }^{131}$ was given to nearly all BIR patients $(96.4 \%)$. As expected from their higher ATA risk score, the initial $\mathrm{I}^{131}$ dose was higher for the BIR-to-SIR patients $(p=0.028)$. While no recurrences were recorded for the BIR patients, structural recurrence in the BIR-to-SIR group was local in $50 \%$ of the patients, LN was found in in $26.5 \%$ of patients, and distant metastases (DM) were found in $42.4 \%$ of patients, which resulted in re-operation $(44.1 \%)$, additional I ${ }^{131}$ therapy $(97.1 \%)$, and external beam radiotherapy $(23.5 \%)$. Interestingly, driven by the persistent thyroglobulin levels, additional $\mathrm{I}^{131}$ therapy was given to $58.7 \%$ of BIR patients as well. Nevertheless, in the BIR-to-SIR group, the cumulative I ${ }^{131}$ dose was $350 \mathrm{mCi}$ compared to $180 \mathrm{mCi}$ in the BIR group $(p=0.001)$.

At the end of the study, the all-cause mortality rate was $10.8 \%$, while disease-related mortality was $3.6 \%$, both of which were entirely in the BIR-to-SIR group $(3 / 34,8.8 \%)$. All three patients had distant metastases to the lungs, and one of them had a Tall Cell variant of PTC. Overall disease-free survival is shown in Figure 1. 
Table 3. Additional treatment and follow-up.

\begin{tabular}{lcccc}
\hline \multicolumn{1}{c}{ Variable } & All & BIR-to-SIR & BIR/Cure & $p$ Value \\
\hline$n$ & 83 & 34 & 49 & - \\
Local recurrence, $n(\%)$ & $17(20.5 \%)$ & $17(50.0 \%)$ & - & - \\
LN recurrence, $n(\%)$ & $9(10.8 \%)$ & $9(26.5 \%)$ & - & - \\
Distant metastases, $n(\%)$ & $14(16.9 \%)$ & $14(42.4 \%)$ & - & - \\
Re-operation, $n(\%)$ & $16(19.3 \%)$ & $15(44.1 \%)$ & - & 0.001 \\
Additional I $131, n(\%)$ & $60(75.0 \%)$ & $33(97.1 \%)$ & $27(58.7 \%)$ & $<0.001$ \\
I $^{131}$ cumulative dose, median & 288 & $350(300,450)$ & $180(150,300)$ & \\
$(\mathrm{mCi})$ & $(162.5350)$ & & & $<0.001$ \\
External beam radiotherapy, & $9(11.0 \%)$ & $8(23.5 \%)$ & $1(2.1 \%)$ & 0.180 \\
$n(\%)$ & $9(10.8 \%)$ & $7(20.6 \%)$ & $2(4.1 \%)$ & 0.104 \\
Death, $n(\%)$ & $3(3.6 \%)$ & $3(8.8 \%)$ & $0(0 \%)$ & \\
Disease-related deaths $n(\%)$ & & &
\end{tabular}

LN, lymph node.

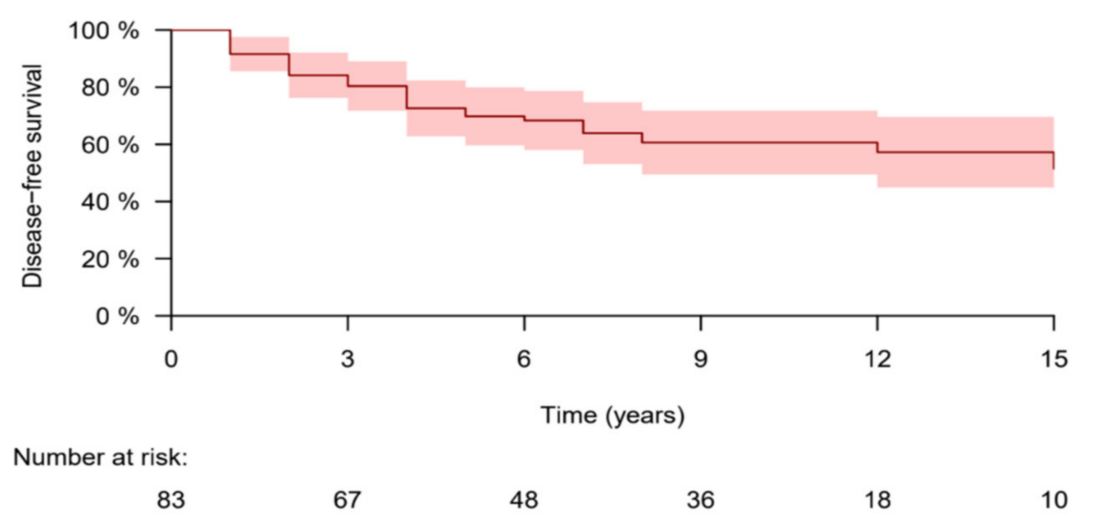

Figure 1. Overall disease-free survival for biochemically persistent DTC patients.

\section{Discussion}

Biochemical incomplete response to treatment at the first two years postoperatively does not always imply that the patient has a persistent thyroid cancer. In some cases, an incomplete thyroidectomy results in persistent low level Tg values arising from nonmalignant thyrocytes. To identify those patients with true BIR, clinicians need to integrate the primary static assessment with prognostic factors during follow-up, which will be discussed here. BIR is observed among 11-19\% of low-risk, 21-22\% of intermediate-risk, and $16-18 \%$ of high-risk patients as per the ATA [2]. In newer publications, BIR is observed at about $15-20 \%$ in all risk categories, with decreasing ER and increasing SIR according to risk category [4,5]. We analyzed 83 patients from a cohort of 1049 (7.9\%) with a biochemical incomplete response throughout a mean follow-up of $12.0 \pm 6.6$ years. During the followup $59.0 \%$ reverted to NED or remained BIR, while $41.0 \%$ progressed to structural disease.

\subsection{Tg Levels and Timing Definition}

There are variations in both the time interval of TG level testing and in the cutoff levels between ER and BIR. According to the ATA, BIR is defined as negative imaging and suppressed $\mathrm{Tg} \geq 1 \mathrm{ng} / \mathrm{mL}$ or Stimulated $\mathrm{Tg} \geq 10 \mathrm{ng} / \mathrm{mL}$ or rising anti-Tg antibody levels. While the specific cutoff levels of Tg that optimally distinguish normal residual thyroid tissue from persistent thyroid cancer have not been determined, rising $\mathrm{Tg}$ values over time are suspicious for growing thyroid tissue or cancer. Most authors regard $\mathrm{Tg}<1 \mathrm{ng} / \mathrm{mL}$ as ER, while others refer to stimulated $\mathrm{Tg}<2 \mathrm{mg} / \mathrm{mL}$ as the cutoff between ER and BIR [6]; some use stimulated Tg [7-9], and others use suppressed or trends in anti Tg Ab titers. The time interval for $\mathrm{Tg}$ level testing varies, potentially starting from 8-12 months after initial therapy [10] to 2 years post initial therapy [2]. The response-to-treatment assessment interval does also differ between studies, ranging from 6 to 24 months after 
initial therapy [1]. Our group measured Tg levels at 1 year postoperatively, and the last visit was adopted as the ATA cutoff point. Nevertheless, we did not find a significant difference in the $\mathrm{Tg}$ levels (either stimulated or suppressed) among the patients who developed structural recurrence compared to patients who remained BIR or who reverted to NED. Of note, only a small subset of patients had enough serial $\mathrm{Tg}$ measurements available to address the doubling time as a possible predictor [11-13]. Applying dynamic risk stratification based on post treatment $\mathrm{Tg}$ levels only revealed that a minority of BIR patients will eventually develop a structural disease, and the $\mathrm{Tg}$ trend was found to be useful in identifying those patients $[14,15]$. Nevertheless, additional treatments do not invariably achieve a biochemical cure, which is partly due to highly sensitive laboratory tests $[3,9,16,17]$, nor do additional treatments prevent the shift from BIR to SIR.

\subsection{Age as a Prognostic Factor for Structural Recurrence}

Response to treatment is associated with patient age: according to the ATA, the ER rate among high-risk patients is significantly higher among younger $(<55$ year) patients, while SIR is significantly higher among older patients [1]. Shah et al. [18] showed that high-risk (HR) patients who are younger than 55 years of age at diagnosis will have twice the chance of achieving an excellent response when compared to older patients. Kim et al. [19] found more gene mutations in patients older than 55 years of age at diagnosis, which was associated with more aggressive pathways compared to younger patients. In line with previous reports [20-22], our data show that age does not predict the BIR-to-SIR shift.

\subsection{Tumor Characteristics}

The only significant differences between BIR/cure and BIR-to-SIR patients were the ATA risk and advanced TNM stage at the time of diagnosis.

Most of our BIR patients $(72.8 \%$ ) were at a low risk (LR) as per the ATA, while only $8.4 \%$ were high risk (HR). However, the ATA risk in the BIR-to-SIR group was 55.9\% LR and in $14.7 \%$ HR compared to $85.1 \%$ and $4.3 \%$ in the BIR/cure group $(p<0.001)$. Thus, initial ATA risk assessment was a significant predictor for structural recurrence in BIR patients. At diagnosis, $13 / 83$ patients (16.1\%) were at T-stage II/III; $26.4 \%$ were BIR-to-SIR patients, and $8.5 \%$ were in the BIR-to-cure group $(p=0.001)$. Thus, an advanced tumor stage at diagnosis appears to be another prognostic factor for structural recurrence in BIR patients. Similar findings were reported by Vaisman et al. [23], who also reported poor outcomes among HR patients (68\% with persistent/recurrent disease and $16.5 \%$ of death) compared to intermediate-risk ( $35.7 \%$ of persistence and $0.9 \%$ of death) and low-risk patients $(12.5 \%$ of persistence and $0 \%$ of death, $p<0.001)$. In his study, only $15.5 \%$ of the HR patients achieved NED at the last follow-up, as opposed to $87.5 \%$ of patients in the LR group $(p<0.001)$. We found a disease-related mortality of $3.6 \%$, which was predominantly in the high-risk ATA stage and in the BIR to SIR group; this is in line with others [24,25], demonstrating that the mortality and progression of DTC mostly occur in the structurally incomplete status.

\subsection{Developing Structural Recurrence}

In our cohort, $41 \%$ progressed to structural recurrence, with $50 \%$ of them remaining SIR at last visit. In Vaisman et al., 55.6\% of the BIR patients had NED at the end of follow up. Although patients with BIR generally have a favorable outcome, some authors $[6,26,27]$ have found immunohistochemistry for BRAFV600E positivity as a risk factor for structural recurrence. Zern et al. [6] found that $34.4 \%$ of BIR patients developed a structural recurrence and that all of these patients were associated with BRAFV600E positive tumors. On the other hand, Ito et al. [28] found that patients with BRAFV600E were equally distributed amongst both high- and low-risk cases. So far, no single marker can predict clinical outcome or structural recurrence [29], and thorough disease surveillance is required for BIR patients $[25,30]$. 


\subsection{Limitations}

Our study has several limitations. Patient data were collected from different medical centers, as some had therapy completed outside of our center; consequently, some data were missing. To fully assess the long-term risk of recurrence, patients need to have a standardized follow-up for many years; however, some of our patients were lost to follow up, which is not uncommon after achieving ER, and were relocated to another hospital or had their follow-up continued in community clinics. The lack of serial $\mathrm{Tg}$ measurements precluded the use of $\mathrm{Tg}$ slope in the prediction analysis. Regarding histological definitions, new classifications were not applied in our cases and could have been included as differentiated thyroid cancers, such as non-invasive follicular thyroid neoplasm with papillary-like nuclear features (NIFTP) or papillary tumor capsule integrity [31]. On the other hand, the strengths of our study were a large cohort of 1049 patients with a long follow up of 12 years.

\section{Conclusions}

Well-differentiated thyroid cancer patient care has evolved from having an identical treatment and follow-up for all patients to an individualized treatment approach based on dynamic risk stratification. Although many BIR patients will eventually shift to NED, some will progress to structural disease; therefore, they should be followed according to their risk of recurrence (RR) and their serial Tg measurement trends, with varying degree of suspicion based on their initial ATA risk stratification and TNM stage.

Author Contributions: Conceptualization C.B., M.S. and L.M.K.; methodology L.M.K. and Y.M.; formal analysis Y.M. and L.M.K.; data curation L.M.K. and Y.M.; writing - original draft preparation L.M.K., M.S. and Y.M.; writing-review and editing C.B., J.P. and S.K. All authors have read and agreed to the published version of the manuscript.

Funding: This research received no external funding.

Institutional Review Board Statement: This study was conducted according to the guidelines of the Declaration of Helsinki and was approved by the Ethics Committee of Shamir (Assaf Harofeh) Medical Center, Zerifin (protocol code 0072-16, 24 June 2016).

Informed Consent Statement: Patient consent was waived due to the retrospective nature of this study.

Data Availability Statement: The data presented in this study are available on request from the corresponding author. The data are not publicly available due to institutional review board restrictions.

Acknowledgments: The authors thank Sonia Mendlovic, Director of the Department of Pathology in Shamir (Assafh Harofeh) Medical Center, Zerifin, Israel, for her contributions.

Conflicts of Interest: The authors declare no conflict of interest.

\section{References}

1. Haugen, B.R.; Alexander, E.K.; Bible, K.C.; Doherty, G.M.; Mandel, S.J.; Nikiforov, Y.E.; Pacini, F.; Randolph, G.W.; Sawka, A.M.; Schlumberger, M.; et al. 2015 American Thyroid Association Management Guidelines for Adult Patients with Thyroid Nodules and Differentiated Thyroid Cancer: The American Thyroid Association Guidelines Task Force on Thyroid Nodules and Differentiated Thyroid Cancer. Thyroid 2016, 26, 1-133. [CrossRef]

2. Tuttle, R.M.; Tala, H.; Shah, J.; Leboeuf, R.; Ghossein, R.; Gonen, M.; Brokhin, M.; Omry, G.; Fagin, J.A.; Shaha, A. Estimating risk of recurrence in differentiated thyroid cancer after total thyroidectomy and radioactive iodine remnant ablation: Using response to therapy variables to modify the initial risk estimates predicted by the new American thyroid association Staging System. Thyroid 2010, 20, 1341-1349. [CrossRef]

3. Tuttle, R.M. Optimal management of a biochemical incomplete response to therapy in differentiated thyroid cancer: Aggressive treatment or cautious observation? Endocrine 2014, 46, 363-364. [CrossRef] [PubMed]

4. Tuttle, R.M.; Alzahrani, A.S. Risk stratification in differentiated thyroid cancer: From detection to final follow-up. J. Clin. Endocrinol. Metab. 2019, 104, 4087-4100. [CrossRef]

5. Krajewska, J.; Chmielik, E.; Jarzab, B. Dynamic risk stratification in the follow-up of thyroid cancer: What is still to be discovered in 2017? Endocr. Relat. Cancer 2017, 24, R387-R402. [CrossRef] 
6. Zern, N.K.; Clifton-Bligh, R.; Gill, A.J.; Aniss, A.; Sidhu, S.; Delbridge, L.; Learoyd, D.; Robinson, B.; Sywak, M. Disease Progression in Papillary Thyroid Cancer with Biochemical Incomplete Response to Initial Therapy. Ann. Surg. Oncol. 2017, 24, 2611-2616. [CrossRef]

7. Shen, F.-C.; Hsieh, C.-J.; Huang, I.-C.; Chang, Y.-H.; Wang, P.-W. Dynamic Risk Estimates of Outcome in Chinese Patients with Well-Differentiated Thyroid Cancer After Total Thyroidectomy and Radioactive Iodine Remnant Ablation. Thyroid 2017, 27, 531-536. [CrossRef]

8. Lamartina, L.; Montesano, T.; Trulli, F.; Attard, M.; Torlontano, M.; Bruno, R.; Meringolo, D.; Monzani, F.; Tumino, S.; Ronga, G.; et al. Papillary thyroid carcinomas with biochemical incomplete or indeterminate responses to initial treatment: Repeat stimulated thyroglobulin assay to identify disease-free patients. Endocrine 2016, 54, 467-475. [CrossRef] [PubMed]

9. Fabián, P.; Erika, A.; Hernán, T.; Fernanda, B.; Carolina, U.; Graciela, C. Biochemical persistence in thyroid cancer: Is there anything to worry about? Endocrine 2014, 46, 532-537. [CrossRef] [PubMed]

10. Castagna, M.G.; Maino, F.; Cipri, C.; Belardini, V.; Theodoropoulou, A.; Cevenini, G.; Pacini, F. Delayed risk stratification, to include the response to initial treatment (surgery and radioiodine ablation), has better outcome predictivity in differentiated thyroid cancer patients. Eur. J. Endocrinol. 2011, 165, 441-446. [CrossRef] [PubMed]

11. Albano, D.; Tulchinsky, M.; Dondi, F.; Mazzoletti, A.; Lombardi, D.; Bertagna, F.; Giubbini, R. Thyroglobulin doubling time offers a better threshold than thyroglobulin level for selecting optimal candidates to undergo localizing [18F] FDG PET/CT in non-iodine avid differentiated thyroid carcinoma. Eur. J. Nucl. Med. Mol. Imaging 2020, 48, 461-468. [CrossRef]

12. Zhang, X.; Higuchi, T.; Tomonaga, H.; Lamid-Ochir, O.; Bhattarai, A.; Nguyen-Thu, H.; Taketomi-Takahashi, A.; Hirasawa, H.; Tsushima, Y. Early detection of progressive disease using thyroglobulin doubling-time in metastatic differentiated thyroid carcinoma treated with radioactive iodine. Nucl. Med. Commun. 2020, 41, 350-355. [CrossRef]

13. Rössing, R.M.; Jentzen, W.; Nagarajah, J.; Bockisch, A.; Görges, R. Serum Thyroglobulin Doubling Time in Progressive Thyroid Cancer. Thyroid 2016, 26, 1712-1718. [CrossRef]

14. Malandrino, P.; Tumino, D.; Russo, M.; Marescalco, S.; Fulco, R.A.; Frasca, F. Surveillance of patients with differentiated thyroid cancer and indeterminate response: A longitudinal study on basal thyroglobulin trend. J. Endocrinol. Investig. 2019, 42, 1223-1230. [CrossRef] [PubMed]

15. Pitoia, F.; Jerkovich, F. Dynamic risk assessment in patients with differentiated thyroid cancer. Endocr. Relat. Cancer 2019, 26, R553-R566. [CrossRef] [PubMed]

16. Onuma, A.; Beal, E.; Nabhan, F.; Hughes, T.; Farrar, W.B.; Phay, J.; Ringel, M.D.; Kloos, R.T.; Shirley, L.A. Long-Term Efficacy of Lymph Node Reoperation for Persistent Papillary Thyroid Cancer: 13-Year Follow-Up. Ann. Surg. Oncol. 2019, 26, 1737-1743. [CrossRef] [PubMed]

17. Han, J.M.; Kim, W.B.; Yim, J.H.; Kim, T.Y.; Ryu, J.-S.; Gong, G.; Sung, T.-Y.; Yoon, J.H.; Shong, Y.K.; Kim, E.Y. Long-term clinical outcome of differentiated thyroid cancer patients with undetectable stimulated thyroglobulin level one year after initial treatment. Thyroid 2012, 22, 784-790. [CrossRef]

18. Shah, S.; Boucai, L. Effect of age on response to therapy and mortality in patients with thyroid cancer at high risk of recurrence. $J$. Clin. Endocrinol. Metab. 2018, 103, 689-697. [CrossRef]

19. Kim, K.; Kim, J.H.; Park, I.S.; Rho, Y.S.; Kwon, G.H.; Lee, D.J. The Updated AJCC/TNM Staging System for Papillary Thyroid Cancer (8th Edition): From the Perspective of Genomic Analysis. World J. Surg. 2018, 42, 3624-3631. [CrossRef]

20. Pitoia, F.; Jerkovich, F.; Smulever, A.; Brenta, G.; Bueno, F.; Cross, G. Should Age at Diagnosis Be Included as an Additional Variable in the Risk of Recurrence Classification System in Patients with Differentiated Thyroid Cancer. Eur. Thyroid J. 2017, 6, 160-166. [CrossRef]

21. Orlov, S.; Orlov, D.; Shaytzag, M.; Dowar, M.; Tabatabaie, V.; Dwek, P.; Yip, J.; Hu, C.; Freeman, J.L.; Walfish, P.G. Influence of age and primary tumor size on the risk for residual/recurrent well-differentiated thyroid carcinoma. Head Neck 2009, 31, 782-788. [CrossRef] [PubMed]

22. Jukkola, A.; Bloigu, R.; Ebeling, T.; Salmela, P.; Blanco, G. Prognostic factors in differentiated thyroid carcinomas and their implications for current staging classifications. Endocr. Relat. Cancer 2004, 11, 571-579. [CrossRef]

23. Vaisman, F.; Momesso, D.; Bulzico, D.A.; Pessoa, C.H.C.N.; Dias, F.; Corbo, R.; Vaisman, M.; Tuttle, R.M. Spontaneous remission in thyroid cancer patients after biochemical incomplete response to initial therapy. Clin. Endocrinol. 2012, 77, 132-138. [CrossRef] [PubMed]

24. Mukhtar, N.; Aljamei, H.; Aljomaiah, A.; Moria, Y.; Alzahrani, A.S. Natural Course of the American Thyroid Association Response to Therapy Statuses (Dynamic Risk Stratification) in Differentiated Thyroid Cancer. Eur. Thyroid J. 2021, 10, 198-207. [CrossRef]

25. Ahn, J.; Song, E.; Kim, W.G.; Kim, T.Y.; Kim, W.B.; Shong, Y.K.; Jeon, M.J. Long-term clinical outcomes of papillary thyroid carcinoma patients with biochemical incomplete response. Endocrine 2020, 67, 623-629. [CrossRef]

26. Enumah, S.; Fingeret, A.; Parangi, S.; Dias-Santagata, D.; Sadow, P.M.; Lubitz, C.C. BRAF V600E Mutation is Associated with an Increased Risk of Papillary Thyroid Cancer Recurrence. World J. Surg. 2020, 44, 2685-2691. [CrossRef] [PubMed]

27. Prescott, J.D.; Sadow, P.M.; Hodin, R.A.; Le, L.P.; Gaz, R.D.; Randolph, G.W.; Stephen, A.E.; Parangi, S.; Daniels, G.H.; Lubitz, C.C. BRAFV600E status adds incremental value to current risk classification systems in predicting papillary thyroid carcinoma recurrence. Surgery 2012, 152, 984. [CrossRef]

28. Ito, Y.; Yoshida, H.; Kihara, M.; Kobayashi, K.; Miya, A.; Miyauchi, A. BRAFV600E mutation analysis in papillary thyroid carcinoma: Is it useful for all patients? World J. Surg. 2014, 38, 679-687. [CrossRef] 
29. Russo, M.; Malandrino, P.; Nicolosi, M.L.; Manusia, M.; Marturano, I.; Trovato, M.A.; Pellegriti, G.; Frasca, F.; Vigneri, R. The BRAFV600E Mutation Influences the Short- and Medium-Term Outcomes of Classic Papillary Thyroid Cancer, But Is Not an Independent Predictor of Unfavorable Outcome. Thyroid 2014, 24, 1267-1274. [CrossRef] [PubMed]

30. Seejore, K.; Mulla, O.; Gerrard, G.E.; Gill, V.M.; Al-Qaissi, A.; Moor, J.W.; Murray, R.D. Outcomes of 756 patients with differentiated thyroid cancer and excellent response to treatment: An evidence-based paradigm for long-term surveillance strategies. Clin. Endocrinol. 2021. [CrossRef]

31. Giani, C.; Torregrossa, L.; Ramone, T.; Romei, C.; Matrone, A.; Molinaro, E.; Agate, L.; Materazzi, G.; Piaggi, P.; Ugolini, C.; et al. Whole Tumor Capsule Is Prognostic of Very Good Outcome in the Classical Variant of Papillary Thyroid Cancer. J. Clin. Endocrinol. Metab. 2021, 106, e4072-e4083. [CrossRef] [PubMed] 